\title{
Histone methyltransferases regulate the transcriptional expression of ERa and the proliferation of tamoxifen-resistant breast cancer cells
}

\author{
Seung-Su Kim ${ }^{1} \cdot$ Min-Ho Lee ${ }^{1} \cdot$ Mi-Ock Lee ${ }^{1} \mathbb{C}$
}

Received: 21 August 2019 / Accepted: 26 December 2019 / Published online: 2 January 2020

(c) The Author(s) 2020

\begin{abstract}
Purpose Although tamoxifen remains the frontline treatment for ER $\alpha$-positive breast cancers, resistance to this drug limits its clinical efficacy. Most tamoxifen-resistant patients retain ER $\alpha$ expression which may support growth and progression of breast cancers. Therefore, we investigated epigenetic regulation of ER $\alpha$ that may provide a rationale for targeting ER $\alpha$ in these patients.

Methods Expression levels of the mixed-lineage leukemia (MLL) family of proteins in tamoxifen-resistant breast cancer cells and publicly available breast cancer patient data sets were analyzed. Histone methylation levels in $E R \alpha$ promoter regions were assessed using chromatin immunoprecipitation. Expression levels of ER $\alpha$ and its target gene were analyzed using western blotting and real-time qPCR. Cell-cycle was analyzed by flow cytometry.

Results The expression of MLL3 and SET-domain-containing 1A (SET1A) were increased in tamoxifen-resistant breast cancers. An MLL3 chromatin immunoprecipitation-sequencing data analysis and chromatin immunoprecipitation experiments for MLL3 and SET1A suggested that these proteins bound to enhancer or intron regions of the ESR1 gene and regulated histone H3K4 methylation status. Depletion of MLL3 or SET1A downregulated the expression level of ER $\alpha$ and inhibited the growth of tamoxifen-resistant breast cancer cells. Additional treatment with fulvestrant resulted in a synergistic reduction of ER $\alpha$ levels and the growth of the cells.

Conclusions The enhanced expression of MLL3 and SET1A in tamoxifen-resistant breast cancer cells supported the ER $\alpha$ dependent growth of these cells by increasing ER $\alpha$ expression. Our results suggest that targeting these histone methyltransferases might provide an attractive strategy to overcome endocrine resistance.
\end{abstract}

Keywords Breast cancer $\cdot$ Tamoxifen resistance $\cdot$ MLL3 $\cdot$ SET1A $\cdot$ ER $\alpha$

\section{Introduction}

Breast cancer is the most prevalent cancer in women and is one of the leading causes of cancer death in women worldwide [1]. Approximately $70 \%$ of breast cancers express the estrogen receptor (ER) and depend on ER signaling for their growth and progression [2]. By blocking estrogen binding to the ER and, thus, blocking the receptor's action, tamoxifen

Electronic supplementary material The online version of this article (https://doi.org/10.1007/s10549-019-05517-0) contains supplementary material, which is available to authorized users.

Mi-Ock Lee

molee@snu.ac.kr

1 College of Pharmacy and Bio-MAX Institute, Research Institute of Pharmaceutical Sciences, Seoul National University, 1 Gwanak-ro, Gwanak-gu, Seoul 08826, Korea has proven its efficacy and remains the frontline treatment for patients with ER-positive breast cancers, especially in premenopausal women [3]. Nonetheless, intrinsic and acquired resistance to tamoxifen limits its clinical efficacy. Almost half of patients with advanced disease do not respond to the drug and almost all patients with metastatic disease eventually develop resistance to it $[3,4]$. Intriguingly, the majority of patients who have relapsed on tamoxifen treatment retain ER $\alpha$ expression [5, 6]. Several preclinical studies targeting $E R \alpha$ in tamoxifen-resistant cancers have yielded promising results [7-9]. Moreover, fulvestrant, a selective ER degrader, has shown clinical benefits in patients whose cancers have relapsed on tamoxifen, indicating that ER $\alpha$ might continue to play important roles in tamoxifen-resistant breast cancer growth [10-12]. Therefore, the delineation of novel mechanisms of regulation of transcriptional expression of ER $\alpha$ 
might provide therapeutic strategies to overcome tamoxifen resistance in ER-positive breast cancers.

Recent advances in genome-sequencing techniques have elucidated the wide distribution of epigenetic marks and mutations in DNA methyltransferases and histone-modifying enzymes, suggesting a direct link between alterations in the epigenome and cancer [13]. In particular, alterations in the levels of histone H3 lysine 4 (H3K4) methylation, which marks transcriptionally active sites, are generally associated with poor prognosis in renal, liver, and breast cancers [14-16]. Correspondingly, the mixed-lineage leukemia (MLL) family of proteins, MLL1, MLL2, MLL3, MLL4, SET-domain-containing 1A (SET1A), and SET1B, which all have the H3K4-methylating SET-domain, are frequently mutated in various cancers, including those of the breast, lung, large intestines, endometrium, and bladder [17]. In breast cancer tissues, comprehensive DNA sequencing revealed that the genes encoding MLL3 (KMT2C) and MLL4 (KMT2D) were two of the most frequently mutated cancer driver genes $[18,19]$. SET1A is overexpressed in more than $10 \%$ of all breast cancer patients and modulates the proliferation and metastasis of breast cancer cells by regulating p53 target genes, such as ARID3A, SESN1, and TP53INP1, and by regulating a group of matrix metalloproteinases [20-22].

Several reports have demonstrated that the epigenetic control of estrogen-dependent transcription by the MLLfamily of proteins promotes the progression of breast cancer [23-25]. Recent studies have highlighted the importance of these proteins by showing that MLL3 and MLL4 bind to the FOXA1 and ER $\alpha$ proteins in ER-positive breast cancer cells to control ER target gene transcription and proliferation [26, 27]. Furthermore, Manso et al. reported that genetic alterations leading to changes in MLL3 were enriched in tumor samples from patients with breast cancers who relapsed during adjuvant hormonal therapy [28]. Similarly, SET1A was demonstrated to be a factor that is required for the recruitment of TIP60 to ER $\alpha$ target gene promoter regions, and depletion of SET1A decreased estrogen-induced transcription [24]. However, the role of these proteins in tamoxifen resistance is largely unknown. Therefore, we aimed to test whether the proteins of MLL-family are involved in tamoxifen resistance in breast cancer cells.

\section{Methods}

\section{Cell lines}

The human breast cancer cell line MCF7 (HTB-22) and T47D (HTB-133) were obtained from the American Type Culture Collection (Manassas, VA, USA). MCF7 cells were grown in Dulbecco's modified Eagle's (DMEM) medium containing $10 \%$ fetal bovine serum (Hyclone, Logan, UT, USA) and T47D cells were grown in RPMI 1640 medium containing $10 \%$ fetal bovine serum. The tamoxifen-resistant and parent MCF7 sublines (MCF7/TAMR-1, MCF7/TAMR8, and MCF7/S0.5) and T47D sublines (T47D/TR-1, T47D/ TR-2, and T47D/S2) were obtained from Ximbio (London, UK). MCF7/S0.5 cells were maintained in phenol-red-free DMEM:F12 (1:1) containing 1\% fetal bovine serum, $2 \mathrm{mM}$ Glutamax $^{\mathrm{TM}}$ (Gibco-Invitrogen, Carlsbad, CA, USA), and $6 \mathrm{ng} / \mathrm{ml}$ of insulin (Merck KGaA, Darmstadt, Germany). $\mathrm{T} 47 \mathrm{D} / \mathrm{S} 2$ cells were maintained in phenol-red-free RPMI medium:F12 (1:1) containing 2\% fetal bovine serum, $2 \mathrm{mM}$ Glutamax $^{\mathrm{TM}}$, and $8 \mu \mathrm{g} / \mathrm{ml}$ of insulin. Tamoxifen-resistant cell lines were maintained in the media supplemented with $1 \mu \mathrm{M}$ tamoxifen. Whenever $17 \beta$-estradiol $\left(\mathrm{E}_{2}\right)$ was used, the cells were adapted to the media containing $2 \%$ charcoalstripped FBS (Gibco-Invitrogen) for at least $48 \mathrm{~h}$ and the experiments were carried out in the same media. All cells were maintained under $5 \% \mathrm{CO}_{2}$ in humidified air at $37{ }^{\circ} \mathrm{C}$.

\section{Short interfering (si)RNA duplexes and transient transfection}

siRNA duplexes targeting $M L L 1, M L L 2, M L L 3, M L L 4$, SET1A, SET1B, and the gene that encodes the nonspecific green fluorescent protein (GFP) were synthesized and purified by Bioneer Co. Ltd (Daejeon, Korea) (Supplementary Table 1). Transient transfection was performed as described previously [29].

\section{Western blot analysis, reverse transcription-quantitative PCR (RT-qPCR), and chromatin immunoprecipitation (ChIP)}

Western blotting was carried out as described previously using specific antibodies against ER $\alpha$, cyclin D1, c-Myc, histone H3 (Santa Cruz Biotechnology, Dallas, TX, USA), monomethylated histone H3K4 (H3K4me1), dimethylated histone H3K4 (H3K4me2), trimethylated histone H3K4 (H3K4me3) (Abcam, Cambridge, UK), and $\alpha$-tubulin (Merck Millipore, Darmstadt, Germany). Band intensities of each protein were quantified using UVITEC (UVITEC, Cambridge, United Kingdom). RT-qPCR was carried out using specific primers, as described previously [29]. The sequences of the RT-qPCR primer used here are shown in Supplementary Table 1. ChIP assays were performed as described previously using specific antibodies against H3K4me1 and H3K4me3 (Abcam) [29]. The bound target DNA fragments $(152,011,652 \mathrm{~kb}$ to $152,011,781 \mathrm{~kb}$ for ChIP1 and $152,128,814 \mathrm{~kb}$ to $152,128,940 \mathrm{~kb}$ for ChIP2) were detected using PCR. The primers used to amplify DNA fragments are given in the Supplementary Table 1. 


\section{Cell proliferation and cell-cycle analysis}

The proportion of viable cells was assessed on a hemocytometer using trypan blue exclusion. For cell-cycle assays, cells were harvested, fixed in $70 \%$ ethanol, stained with propidium iodide, and analyzed by flow cytometry (Becton Dickinson, Franklin Lakes, NJ, USA).

\section{Chromatin immunoprecipitation-sequencing (ChIP-seq) data analysis}

MLL3 ChIP-seq data and their matched inputs (GSE81714) were downloaded from Gene Expression Omnibus (GEO; https://www.ncbi.nlm.nih.gov/geo/). Enriched regions of the genome were identified by comparing ChIP samples with input samples using the default parameters of the Modelbased Analysis of ChIP-Seq peak-caller tool (v. 1.4.2) [30]. Peaks that were enriched by more than twofold over an input control with a $q$-value of $<0.05$ were selected; 2876 peaks were identified in the MLL3 ChIP-seq data set. Genomewide distributions were generated using the Integrative Genomics Viewer (https://software.broadinstitute.org/softw are/igv/) [31].

\section{Breast cancer patient cohort analysis based on public data sets}

The publicly available data sets GSE9893, GSE3494, and GSE2034 were downloaded from GEO [32-34]. The GSE9893 data set (MLRG Human 21K v. 12.0; Montpellier Génopole Microarray core facility) contains the gene expression profiles of 155 patients treated with tamoxifen for 5 years after surgery. The GSE3494 data set (Affymetrix Human Genome U133A Array) contains the gene expression profiles of 315 patient cohort, among which 213 ERpositive breast cancer patient data was selected for analysis. The GSE2034 data set (Affymetrix Human Genome U133A Array) contains the gene expression profiles of 286 patient cohort, among which 209 ER-positive breast cancer patient data was selected for analysis. The RNA-seq data of The Cancer Genome Atlas (TCGA) breast invasive carcinoma cohort (https://cancergenome.nih.gov/, 601 ER-positive breast cancer patient samples), and the breast cancer patient gene expression profile data of the Netherlands Cancer Institute (NKI-295, 226 ER-positive breast cancer patient samples) were obtained from the Functional Genomics Explorer data bank (https://xena.ucsc.edu/; University of California, Santa Cruz, CA, USA) [35]. The Molecular Taxonomy of Breast Cancer International Consortium (METABRIC) and the GSE42568 data sets were obtained and analyzed in the CTGS website (https://ctgs.biohackers.net/) [36]. The METABRIC dataset contains gene expression profiles of 1980 patient cohort, of which 1398 ER-positive breast cancer patients were selected for analysis. The GSE42568 contains gene expression profiles of 121 patient cohort of which 67 ER-positive breast cancer patients were selected for analysis. No additional manipulation was performed on the data.

\section{Statistical analyses}

Statistical analyses were performed using GraphPad Prism software (GraphPad Software, San Diego, CA, USA). Statistically significant differences between two groups were determined using two-tailed, paired Student's $t$ tests for protein quantification and unpaired Student's $t$ tests for any other analyses. Statistical analyses of multiple groups were performed using a two-way analysis of variance followed by a Bonferroni post-test correction. Data are reported as the mean \pm SEM and $P<0.05$ was considered significant.

\section{Results}

\section{H3K4 methylation is increased in tamoxifen-resistant breast cancer}

To explore the role of epigenetic control in the development of tamoxifen resistance in breast cancer, $\mathrm{H} 3 \mathrm{~K} 4$ methylation levels were measured in the tamoxifen-resistant cells. We observed an increase in mono-, di-, and tri-methylation at H3K4 in the tamoxifen-resistant MCF-7 cells compared with parent cells, whereas $\mathrm{H} 3 \mathrm{~K} 4 \mathrm{me} 3$ is enhanced in tamoxifenresistant T47D cells (Fig. 1a and Supplementary Fig. 1). Because the proteins of the MLL-family are major enzymes that catalyze methylation at $\mathrm{H} 3 \mathrm{~K} 4$ residues, we analyzed the expression of these enzymes in the tamoxifen-resistant cells (Fig. 1b). Consistently, mRNA expression levels of most of the $M L L$-family genes were higher in the tamoxifen-resistant cells. To investigate the clinical relevance of this finding, we analyzed publicly available microarray data sets, which included gene expression profiles obtained from ER-positive breast cancer patients treated with tamoxifen. The expression levels of MLL1, MLL3, MLLA, SET1A, and SETIB were significantly higher in breast cancer tissues from the patients who relapsed compared with those who were relapse free (Fig. 1c) [32]. Moreover, the survival rates analyzed using the Kaplan-Meier method with the log-rank test in two independent public datasets, METABRIC and GSE42568, disease-free survival rate was significantly worse in the high MLL3 or high SET1A expression group (Fig. 1d). These results imply that these $\mathrm{H} 3 \mathrm{~K} 4$ methyltransferases might play a role in the development of tamoxifen resistance in breast cancer. 
Fig. 1 MLL expression is increased in tamoxifen-resistant breast cancer. a, b Total whole cell lysates and RNA obtained from the tamoxifen-resistant cells and their parental cells were subjected to western blotting (a) and qRT-PCR analysis (b). Data presented as mean \pm SEM $(n=3) . * P<0.05$, $* * P<0.01$ and $* * * P<0.001$. c The GSE9893 data set was obtained from NCBI GEO [32]. The expression levels of MLL1, MLL2, MLL3, MLL4, SETIA, and SETIB mRNA in 155 patients with ESR1positive breast cancer treated with tamoxifen for 5 years after surgery are shown by the $\log 2$ expression value. $* P<0.05$ and $* * * P<0.001$. d ER-positive breast cancer patient data from the METABRIC and the GSE42568 data sets were analyzed in the CTGS website (https://ctgs.biohackers.net/) [36]. Patients were categorized into a low gene expression (lower quartile) group and a high gene expression (upper quartile) group. Disease-freesurvival rate (\%) was plotted for each group. To analyze statistical differences, Logrank (Mantel-Cox) tests were performed

A
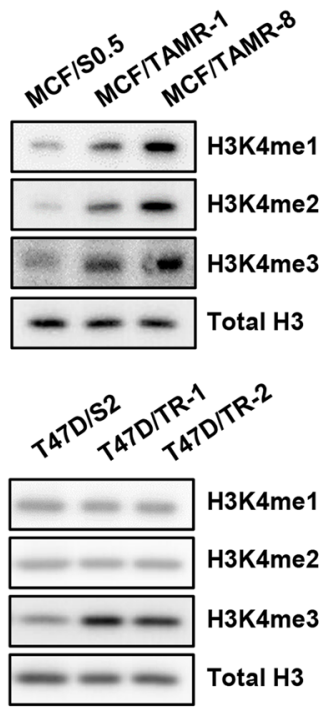

B
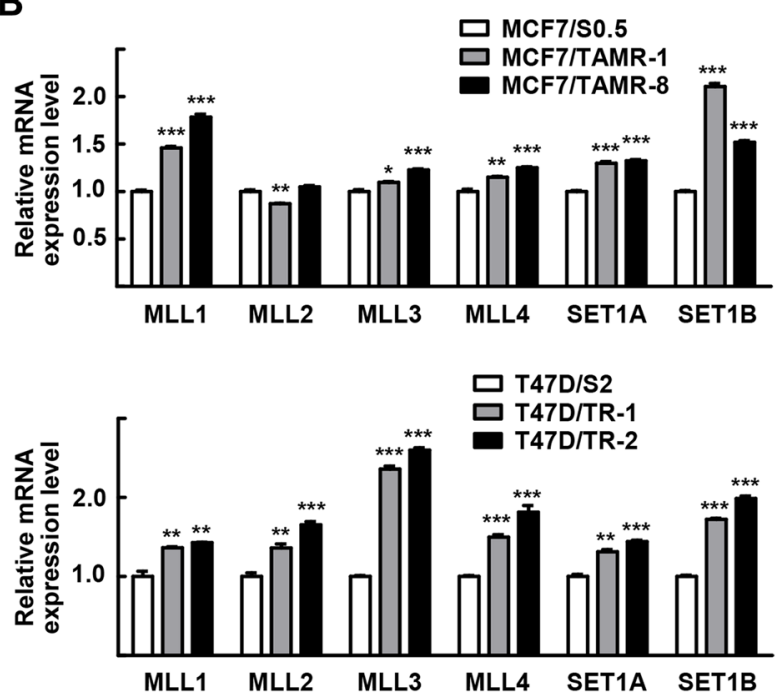

C
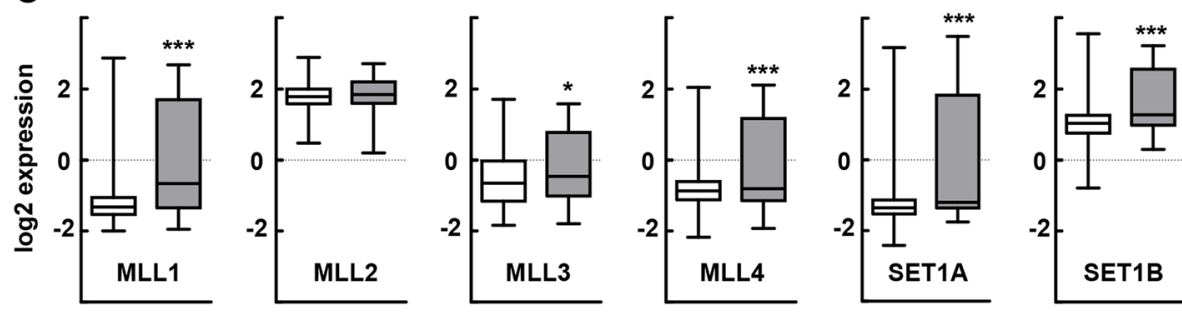

Relapse-free (n=103) $\square$ Relapse (n=52)

D

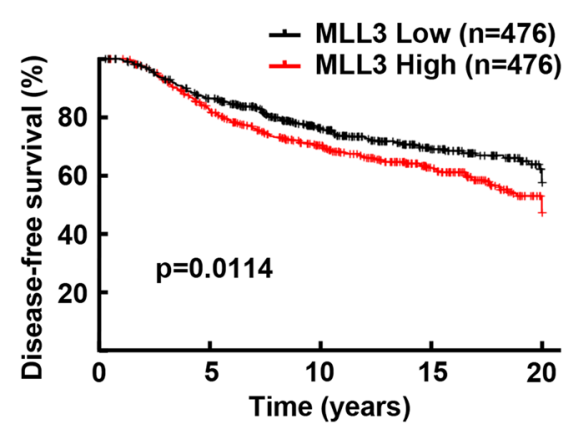

GSE42568

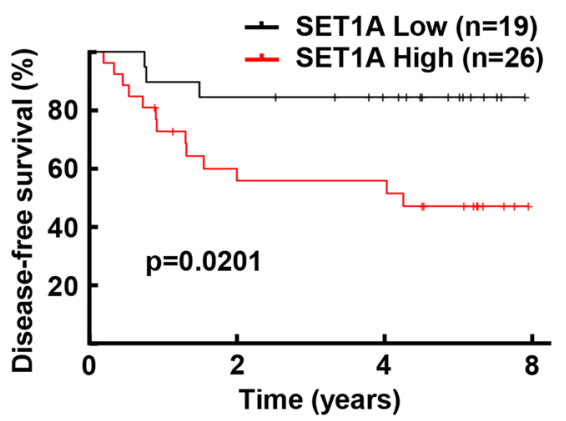

\section{MLL3 and SET1A regulate ERa gene expression}

Tamoxifen-resistant breast cancer tissues and cell lines retain an elevated level of the ER $\alpha$ protein, which may drive cellular growth in these tamoxifen-resistant cells $[6,8,9]$. Therefore, we wondered whether genetic knockdown of the $M L L$-family genes changes $E R \alpha$ gene expression. First, we checked whether genetic knockdown of MLL-family genes affects ER $\alpha$ expression in breast cancer cells, MCF7 and T47D. Among the $M L L$-family genes, knockdown of MLL3 and SET1A resulted in a decrease in the ER $\alpha$ protein and mRNA levels, suggesting the transcriptional control of ER $\alpha$ expression by these histone-modifying enzymes (Fig. 2a, b and Supplementary Fig. 2a, b). The finding that alternative siRNA sequences targeting these genes also downregulated ER $\alpha$ mRNA expression significantly eliminated the possibility of potential off-target effects on ER $\alpha$ mRNA downregulation (Supplementary Fig. 2c).

Next, we explored whether MLL3 and SET1A control the expression of $E R \alpha$ by regulating $E R \alpha$ promoter activity directly in MCF7 cells. We identified the regulatory regions in the ER $\alpha$-encoding genes as potential targets of MLL3 by analyzing recently reported MLL3 ChIPseq data (Fig. 2c) [26]. Four MLL3 binding sites (peaks $1-4)$ were located at the enhancer or intron regions of the ESRI gene; peaks 1-3 were found near exon 1 of the ER $\alpha$ 
A

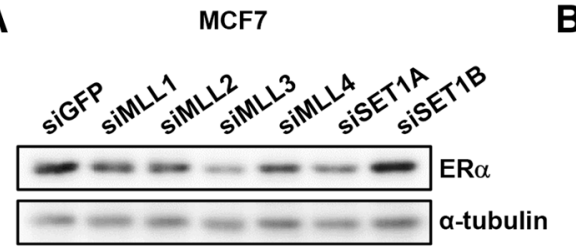

T47D

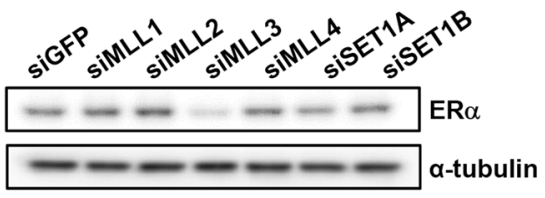

B
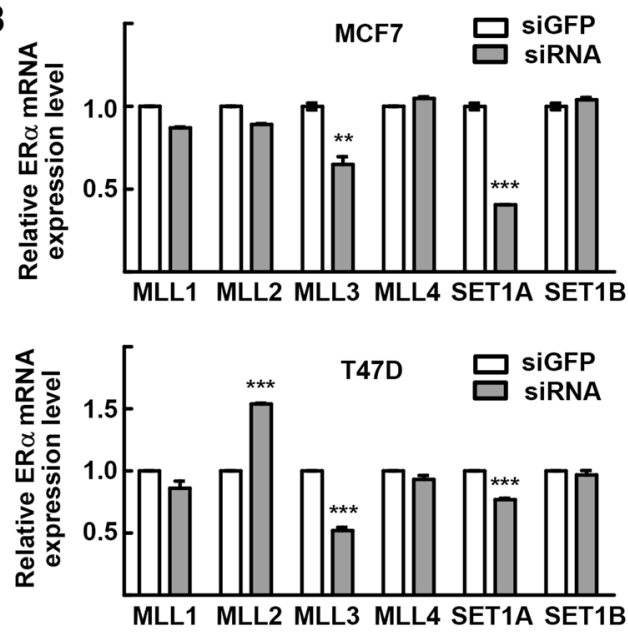

C Chromosome 6 q25.1-q25.2
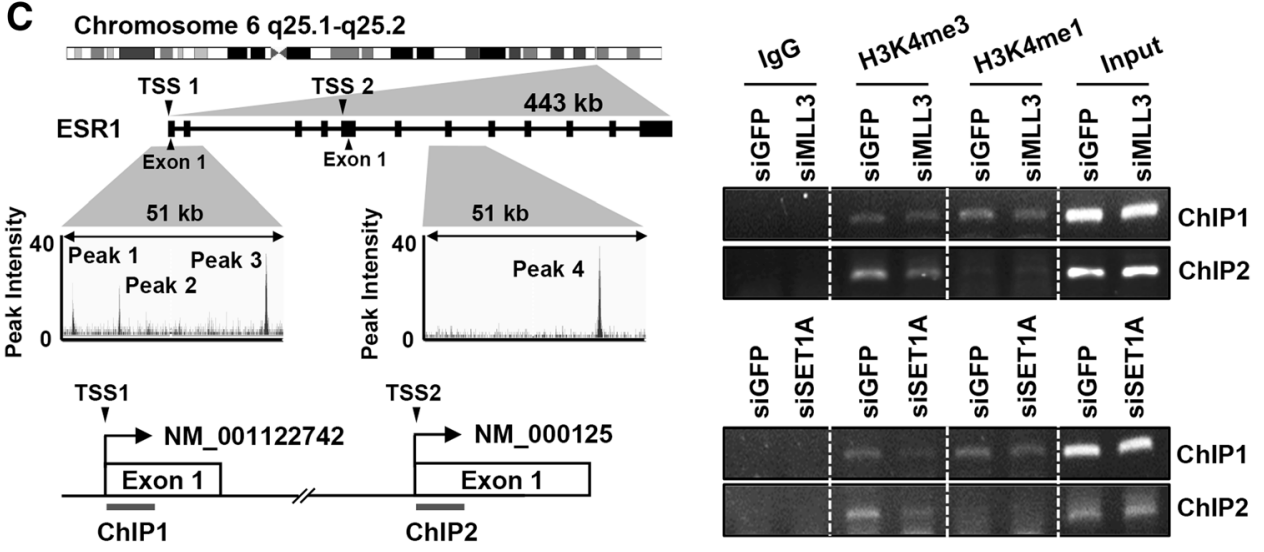

D

TCGA

GSE3494
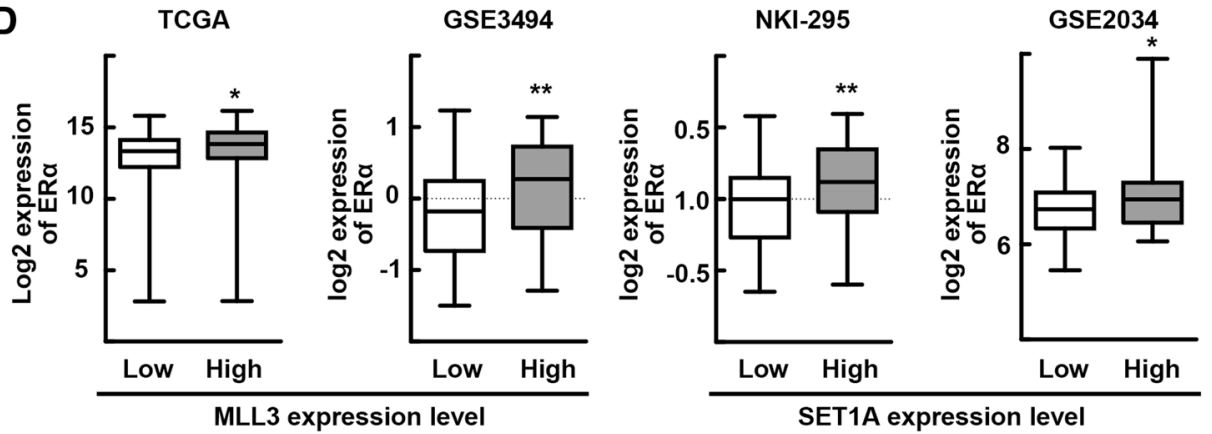

Fig. 2 MLL3 and SET1A regulate ER $\alpha$ gene expression. a, b The MCF7 and T47D cells were transfected with indicated siRNA for $48 \mathrm{~h}$. Total whole cell lysates and RNA obtained from MCF7 and T47D cells were subjected to western blotting (a) and qRT-PCR analysis (b), respectively. Data presented as mean $\pm \operatorname{SEM}(n=3)$. $* * P<0.01$ and $* * * P<0.001$. c Integrative Genomics Viewer tracks showed four MLL3 binding peaks in the ER $\alpha$ promoter/enhancer regions which was generated based on the analysis of the MLL3 ChIP-seq data set (GSE81714). Boxes and lines in the ESR1 gene represent exons and introns, respectively (left top). Schematic representation of the human $\mathrm{ER} \alpha$ promoter/enhancer regions for ChIP experiments (left bottom). MCF7 cells were transfected with siMLL3 or siSET1A for $48 \mathrm{~h}$. DNA fragments that were immunoprecipitated by anti-H3K4me3 or anti-H3K4me1 antibodies were amplified by

PCR using primers for ChIP1 and ChIP2 (right). TSS: transcriptional start site. d The mRNA expression of ER $\alpha$ and MLL3 in ER-positive breast cancer patients are based on TCGA RNA-seq or GSE3494 microarray data set (https://xena.ucsc.edu/) [33]. The mRNA expression of ER $\alpha$ and SET1A in ER-positive breast cancer patients are based on GSE2034 microarray data set or NKI microarray data set (https://xena.ucsc.edu/) [34, 35]. Patients were categorized into a low gene expression (lower quartile) group and a high gene expression (upper quartile) group. TCGA RNA-seq: $n=150$ for MLL3-low and $n=150$ for MLL3-High; GSE3494 microarray: $n=62$ for MLL3-low and $n=62$ for MLL3-High; NKI-295 microarray: $n=56$ for SET1Alow and $n=56$ for SET1A-High; GSE2034 microarray: $n=52$ for SET1A-low and $n=52$ for SET1A-High. $* P<0.05$ and $* * P<0.01$ 
transcript NM_001122742 and peak 4 was located at an intron of the transcript NM_000125. Therefore, we examined whether the $\mathrm{H} 3 \mathrm{~K} 4$ methylation status in the ESR 1 gene was altered by MLL3. ChIP assays were performed using anti-MLL3 antibodies and two sets of PCR primers spanning the first exon of two different ER $\alpha$ transcripts, NM_001122742 and NM_000125. We found that knockdown of MLL3 reduced both H3K4me3 and H3K4me1 in the exon 1 region in both transcripts (ChIP 1 and ChIP 2), although $\mathrm{H} 3 \mathrm{~K} 4 \mathrm{me} 1$ was not detected in the ChIP 2 region (Fig. 2c, Supplementary Fig. 2d). Interestingly, SET1A depletion yielded a similar pattern of histone methylation in the ESRl gene (Fig. 2c, Supplementary Fig. 2d). These results indicate that MLL3 and SET1A may regulate ER $\alpha$ expression by modifying the $E R \alpha$ promoter/enhancer regions via its histone methyltransferase activity. Consistent with these data, ER $\alpha$ mRNA levels in ER-positive breast cancer tissues were significantly higher in the cancer tissues with high expression of MLL3 or SET1A, as assessed based on the analysis of the publicly available breast cancer data sets (Fig. 2d) [33-35].

\section{Knockdown of MLL3 and SET1A decreases expression of ER-dependent growth-associated genes}

Next, we examined whether knockdown of MLL3 or SET1A affected the expression of downstream targets of ER $\alpha$, such as the progesterone receptor $(P R)$, trefoil factor $1(T F F 1)$, and cyclin D1 (CCNDI) genes. The mRNA levels of $E R \alpha$, as well as those of $P R$ and TFF1, were reduced significantly after knockdown of MLL3. Similarly, knockdown of SET1A decreased significantly the mRNA levels of $E R \alpha, P R$, and $C C N D 1$, indicating that both MLL3 and SET1A are critical for the expression of ER $\alpha$ and its target genes (Fig. 3a). Similarly, knockdown of MLL3 and SET1A resulted in the reduced level of ER $\alpha$ and its target genes in both MCF7/S0.5 and MCF7/TAMR-1 cells (Supplementary Fig. 3a). Consistently, knockdown of SET1A reduced the levels of the ER $\alpha$, CCND1, and c-MYC proteins (Fig. 3b). These downregulations were more obvious in the MCF7/TAMR-1 cells compared with their parent cells (Supplementary Fig. 3a, b). These results indicate that MLL3 and SET1A are responsible for the expression of ER $\alpha$ and its target genes, especially in tamoxifen-resistant breast cancer cells.
Fig. 3 MLL3 and SET1A decrease gene expression of $\mathrm{ER} \alpha$ and its downstream target genes and cell-growth. The MCF7, MCF7/S0.5, or MCF7/ TAMR-1 cells were transfected with siMLL3 or siSET1A for $48 \mathrm{~h}$. a Total RNA obtained from the MCF7 cells were subjected to qRT-PCR analysis. Data presented as mean \pm SEM $(n=3) . * P<0.05, * * P<0.01$ and $* * * P<0.001$. b The whole cell lysates obtained from MCF7/S0.5 or MCF7/ TAMR- 1 cells were subjected to western blotting. $\mathbf{c}$ The number of viable cells were counted using a hemocytometer. Cell numbers were presented as the mean \pm SEM from duplicate plates. One of three independent experiments with similar results are presented. $* * * P<0.001$. d Transfected cells were stained with propidium iodide and analyzed by flow cytometry for cell-cycle status

\section{A}
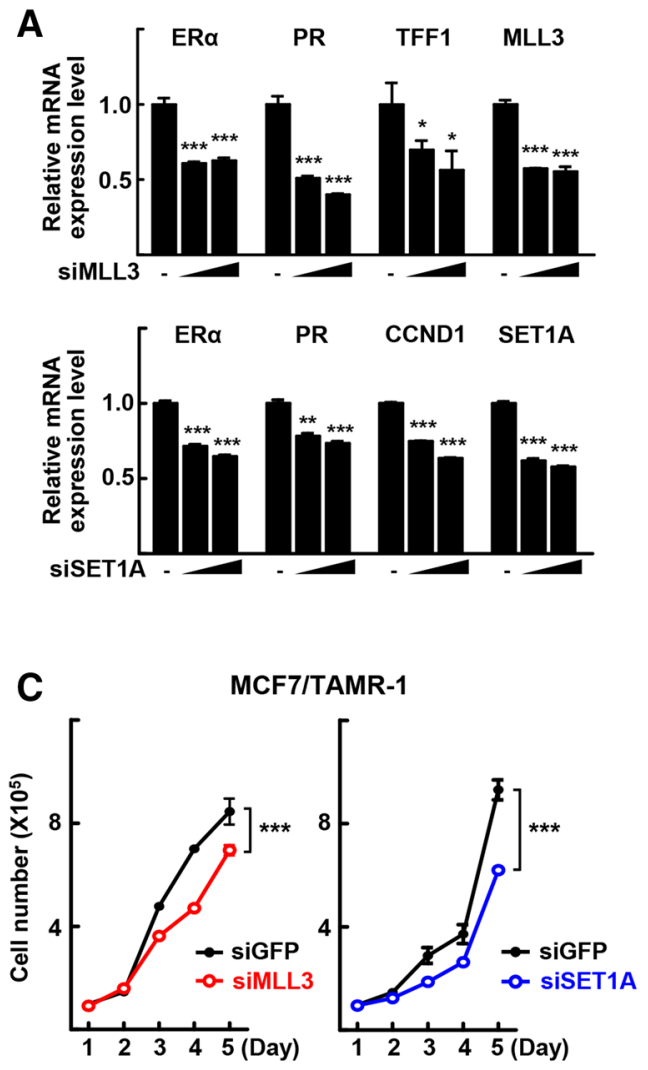

B
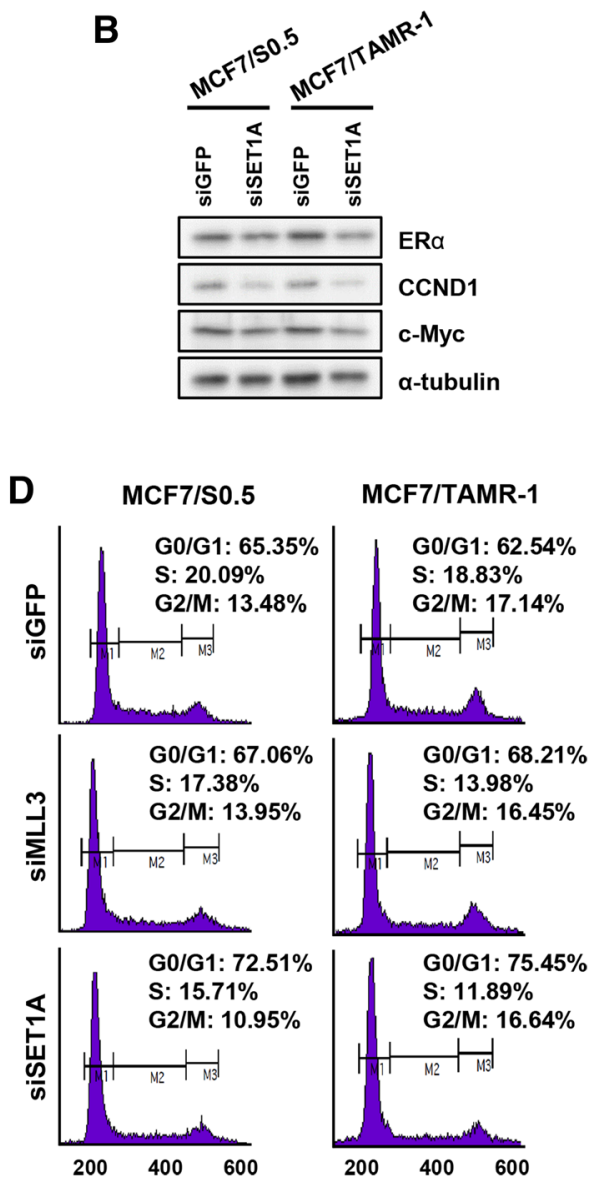
Several clinical trials support the notion that ER $\alpha$ is a pivotal driver of endocrine resistance in breast cancer and continues to be an important therapeutic target $[5,6]$. Therefore, we investigated whether knockdown of MLL3 and SET1A inhibits the growth of the tamoxifen-resistant MCF7/TAMR-1 cells. Knockdown of MLL3 or SET1A decreased the number of MCF7/TAMR- 1 cells by about $20 \%$ or $35 \%$, respectively, after 5 days of culture (Fig. 3c). An $E_{2}$ treatment increased largely the growth of MCF7/TAMR-1 cells, however the $\mathrm{E}_{2}$-induced growth was reduced significantly by knockdown of MLL3 or SET1A (Supplementary Fig. 3c). Although the $E_{2}$ treatment increased the growth of parental cells, knockdown of MLL3 or SET1A affected marginally in the cell-growth regardless of the $\mathrm{E}_{2}$ treatment (Supplementary Fig. 3c). Cell-cycle analysis by flow cytometry showed that knockdown of MLL3 or SET1A increased the G0/G1 phase in MCF7/TAMR-1 cells by almost twofold compared with the control cells (Fig. 3d). These results demonstrated that the expression of ER $\alpha$ is important for the histone methyltransferase-associated inhibition of the growth of tamoxifen-resistant breast cancer cells.

\section{Inhibition of MLL3 and SET1A enhances fulvestrant sensitivity in tamoxifen-resistant cells}

Finally, we evaluated the effect of fulvestrant, an ER $\alpha$ protein degrader, on the MLL3- or SET1A-knockdown-induced downregulation of ER $\alpha$. We expected a further reduction of $E R \alpha$ levels based on two distinct mechanisms of $E R \alpha$ regulation, which may lead to enhanced sensitivity to fulvestrant of tamoxifen-resistant cells [10]. The ER $\alpha$ protein levels were decreased after treatment with fulvestrant in MCF7/TAMR-1 cells and were further decreased by knockdown of MLL3 or SET1A (Fig. 4a). Knockdown of MLL3 or SET1A together with fulvestrant treatment yielded an enhanced inhibition of the growth of MCF7/TAMR-1 cells, suggesting that ER $\alpha$ downregulation causes a reduction in cell-growth signaling (Fig. 4b).

\section{Discussion}

In this study, we report for the first time that the H3K4 methyltransferases MLL3 and SET1A regulate the transcriptional expression of $\mathrm{ER} \alpha$ by altering histone methylation patterns in the $E R \alpha$ promoter regions. In particular, the expression levels of MLL3 and SET1A were enhanced in the cancer tissues of patients with tamoxifen-resistant breast cancer, which may lead to the ER $\alpha$-dependent proliferation of these cells (Figs. 1 and 2).

The histone methyltransferases of the MLL-family are highly conserved across eukaryotes and share the SET-domain, which is responsible for catalyzing histone
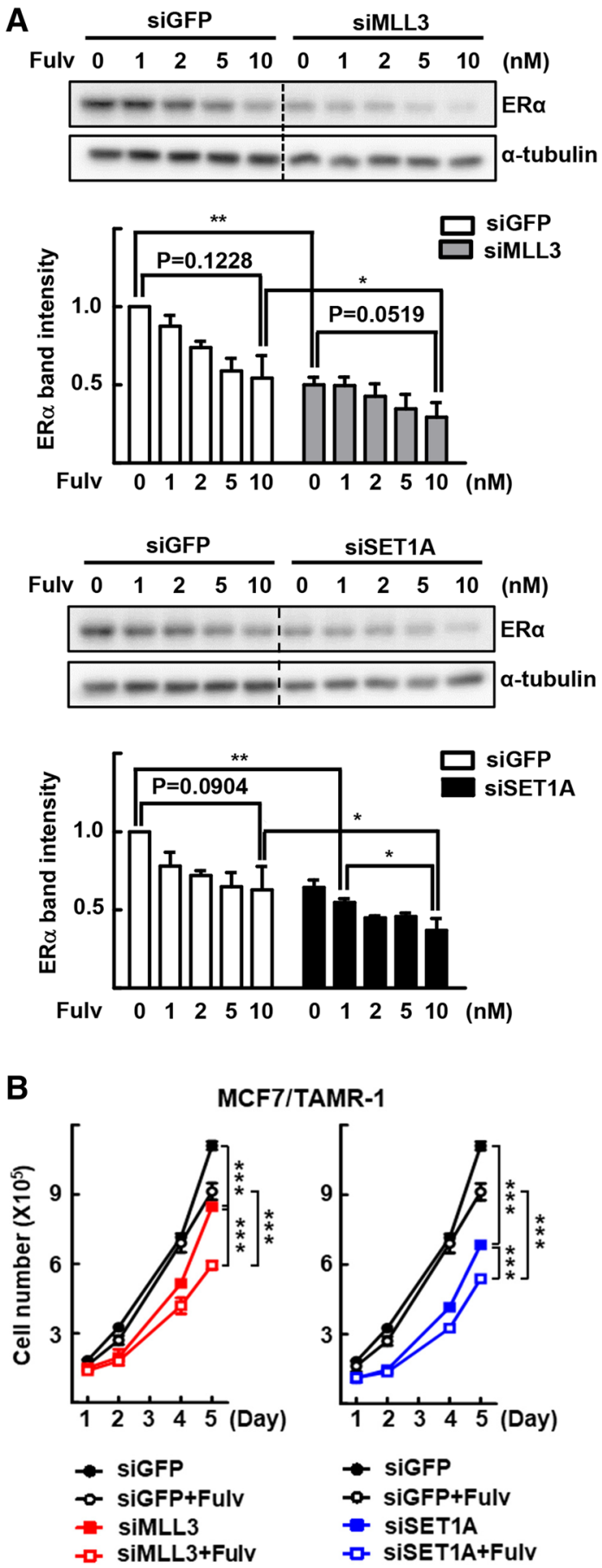

Fig. 4 Inhibition of MLL3 and SET1A expression enhances sensitivity against fulvestrant-induced cell-growth inhibition in tamoxifen-resistant cells. a The MCF7/TAMR-1 cells were transfected with indicated siMLL3 or siSET1A for $48 \mathrm{~h}$. Transfected cells were treated with the indicated concentration of fulvestrant (Fulv) for $24 \mathrm{~h}$. The expression of ER $\alpha$ was determined by western blotting (top). Band intensities of ER $\alpha$ were quantified and normalized to that of $\alpha$-tubulin. Data presented as mean \pm SEM $(n=4)$. $* P<0.05$ and $* * P<0.01$ (bottom). b The MCF7/TAMR-1 cells were transfected with indicated siMLL3 or siSET1A for $48 \mathrm{~h}$. Transfected cells were treated with $5 \mathrm{nM}$ fulvestrant for the indicated time periods and the number of viable cells was counted using a hemocytometer. Cell numbers were presented as the mean \pm SEM from duplicate plates. One of three independent experiments with similar results are presented. $* * * P<0.001$ 
methylation on H3K4 residues [37]. The MLL3 and SET1A members of this family may have redundancy on ER $\alpha$ transcription in breast cancer. For example, both MLL3 and SET1A modulate the histone methylation status near the MLL3 binding sites located in enhancer or intronic regions of the ESRI gene (Fig. 3). In particular, previous reports from our group and others have shown that the proximal region of the $E R \alpha$ promoter, including the exon 1 region in the NM_000125 sequence (ChIP2), is under the control of various epigenetic regulators, such as DNA methyltransferases and histone deacetylases [29, 38-40]. Furthermore, the ER $\alpha$ mRNAs transcribed from these two TSS sites are two of the four major ER $\alpha$ transcripts [41, 42]. The ER $\alpha$ transcript from NM_000125 represents half of the total ER $\alpha$ mRNA, and the ER $\alpha$ transcript from NM_001122742 represents almost $10 \%$ of the total ER $\alpha$ mRNA, which underscores the importance of the epigenetic function of both MLL3 and SET1A. Interestingly, a meta-analysis of 51 histone methyltransferases in 958 breast cancer samples revealed that SET1A is amplified, while MLL3 is mutated the most in the Luminal A subtype, which highlights the significance of MLL3 and SET1A in ER $\alpha$ signaling [43].

Our study, together with recent publications, highlights the important functions of MLL3 and SET1A in ER $\alpha$ signaling at two different levels. First, these histone methyltransferases regulate the transcriptional function of ER $\alpha$ by acting on ER $\alpha$-binding sites. For example, Jozwik et al. showed that MLL3 forms a complex with ER $\alpha$ and FOXA1 in the enhancer regions of $E R \alpha$ target genes, such as $c-M Y C$ and TFF1 [26]. Gala and colleagues also showed that MLL3 is necessary for ER $\alpha$ enhancer function, while its loss reprogrammed ER $\alpha$ to regulate its target genes via an AP-1-dependent mechanism, causing hormone independency [44]. Similar to the function of MLL3 on the transcriptional activity of ER $\alpha$, SET1A facilitated the binding of ER $\alpha$ to the promoter/enhancer regions of ER target genes and increased estrogen-induced transcription [24, 45]. Accordingly, depletion of SET1A decreased the proliferation of ER $\alpha$-positive and tamoxifen-resistant breast cancer cells [45]. Second, we showed here that MLL3 and SET1A upregulated ER $\alpha$ by inducing the active chromatin form on the promoter regions of the ER $\alpha$ gene (Fig. 2). Together, MLL3 and SET1A may act in a dual mode in ER-positive and tamoxifen-resistant breast cancer cells to confer ER-dependent breast cancer cell-growth, which could be therapeutically exploited by employing ER $\alpha$ antagonists, such as fulvestrant (Fig. 4).

Fulvestrant causes ER $\alpha$ protein destruction upon binding and has demonstrated clinical efficacy among patients who relapsed for a second time after responding to tamoxifen and aromatase inhibitors [10-12]. Fulvestrant treatment, as well as knockdown of the $E R \alpha$ gene, reduced the growth of tamoxifen-resistant breast cancer cells; moreover, this strategy was efficacious in several xenograft experiments $[7,46$,
47]. In this study, we demonstrated that the combination of depletion of MLL3 or SET1A and fulvestrant treatment downregulated ER $\alpha$ and inhibited breast cancer cell-growth more effectively (Fig. 4). Therefore, this combination strategy may provide an insight into the regulation of the growth of tamoxifen-resistant breast cancer cells that have acquired alternative means of sustaining growth.

\section{Conclusions}

Here, we report that the H3K4 methyltransferases MLL3 and SET1A regulate ER $\alpha$ expression epigenetically in ER $\alpha$ positive breast cancer cells. We also found that MLL3 and SET1A were upregulated in tamoxifen-resistant breast cancer cells and that their depletion inhibited the expression of the target genes of ER $\alpha$ and repressed the proliferation of these cells. These effects were enhanced by treatment with fulvestrant. Thus, targeting these histone methyltransferases could provide an attractive strategy for overcoming endocrine resistance in patients with breast cancer.

Author contributions SSK, MHL, and MOL contributed to the design of experiments and writing of the manuscript. SSK and MHL performed the experiments. MHL analyzed the ChIP-sequencing data. All authors read and approved the final manuscript.

Funding This project was supported by Grants from the National Research Foundation of Korea (2017R1A2B3011870 and 2018R1A5A2024425), and Korea Mouse Phenotyping Project (NRF-2014M3A9D5A01073556).

Data availability All data generated or analyzed during this study are included in this published article and its supplementary information files. Any additional information is available upon reasonable request to the corresponding author.

\section{Compliance with ethical standards}

Conflict of interest The authors declare that they have no conflict of interest.

Human and animal rights This article does not contain any studies with human participants or animals performed by any of the authors.

Open Access This article is licensed under a Creative Commons Attribution 4.0 International License, which permits use, sharing, adaptation, distribution and reproduction in any medium or format, as long as you give appropriate credit to the original author(s) and the source, provide a link to the Creative Commons licence, and indicate if changes were made. The images or other third party material in this article are included in the article's Creative Commons licence, unless indicated otherwise in a credit line to the material. If material is not included in the article's Creative Commons licence and your intended use is not permitted by statutory regulation or exceeds the permitted use, you will need to obtain permission directly from the copyright holder. To view a copy of this licence, visit http://creativecommons.org/licenses/by/4.0/. 


\section{References}

1. Torre LA, Islami F, Siegel RL et al (2017) Global cancer in women: burden and trends. Cancer Epidemiol Biomark Prev 26(4):444-457. https://doi.org/10.1158/1055-9965.EPI-16-0858

2. Lumachi F, Brunello A, Maruzzo M et al (2013) Treatment of estrogen receptor-positive breast cancer. Curr Med Chem 20(5):596-604

3. Early Breast Cancer Trialists' Collaborative Group, Davies C, Godwin J et al (2011) Relevance of breast cancer hormone receptors and other factors to the efficacy of adjuvant tamoxifen: patient-level meta-analysis of randomised trials. Lancet 378(9793):771-784. https://doi.org/10.1016/S0140 -6736(11)60993-8

4. Nass N, Kalinski T (2015) Tamoxifen resistance: from cell culture experiments towards novel biomarkers. Pathol Res Pract 211(3):189-197. https://doi.org/10.1016/j.prp.2015.01.004

5. Gong Y, Han EY, Guo M et al (2011) Stability of estrogen receptor status in breast carcinoma: a comparison between primary and metastatic tumors with regard to disease course and intervening systemic therapy. Cancer 117(4):705-713. https://doi. org/10.1002/cncr.25506

6. Gutierrez MC, Detre S, Johnston S et al (2005) Molecular changes in tamoxifen-resistant breast cancer: relationship between estrogen receptor, HER-2, and p38 mitogen-activated protein kinase. J Clin Oncol 23(11):2469-2476. https://doi.org/10.1200/ JCO.2005.01.172

7. Feng Q, Zhang Z, Shea MJ et al (2014) An epigenomic approach to therapy for tamoxifen-resistant breast cancer. Cell Res 24(7):809-819. https://doi.org/10.1038/cr.2014.71

8. Kuske B, Naughton C, Moore K et al (2006) Endocrine therapy resistance can be associated with high estrogen receptor alpha (ERalpha) expression and reduced ERalpha phosphorylation in breast cancer models. Endocr Relat Cancer 13(4):1121-1133. https://doi.org/10.1677/erc.1.01257

9. Thrane S, Lykkesfeldt AE, Larsen MS et al (2013) Estrogen receptor alpha is the major driving factor for growth in tamoxifen-resistant breast cancer and supported by HER/ERK signaling. Breast Cancer Res Treat 139(1):71-80. https://doi.org/10.1007/ s10549-013-2485-2

10. Osborne CK, Wakeling A, Nicholson RI (2004) Fulvestrant: an oestrogen receptor antagonist with a novel mechanism of action. Br J Cancer 90(1):S2-S6. https://doi.org/10.1038/sj.bjc.6601629

11. Ingle JN, Suman VJ, Rowland KM et al (2006) Fulvestrant in women with advanced breast cancer after progression on prior aromatase inhibitor therapy: North Central Cancer Treatment Group Trial N0032. J Clin Oncol 24(7):1052-1056. https://doi. org/10.1200/JCO.2005.04.1053

12. Perey L, Paridaens R, Hawle $\mathrm{H}$ et al (2007) Clinical benefit of fulvestrant in postmenopausal women with advanced breast cancer and primary or acquired resistance to aromatase inhibitors: final results of phase II Swiss Group for Clinical Cancer Research Trial (SAKK 21/00). Ann Oncol 18(1):64-69. https://doi.org/10.1093/ annonc/mdl341

13. Jones PA, Issa JP, Baylin S (2016) Targeting the cancer epigenome for therapy. Nat Rev Genet 17(10):630-641. https://doi. org/10.1038/nrg.2016.93

14. Ellinger J, Kahl P, Mertens C et al (2010) Prognostic relevance of global histone $\mathrm{H} 3$ lysine 4 (H3K4) methylation in renal cell carcinoma. Int J Cancer 127(10):2360-2366. https://doi.org/10.1002/ ijc. 25250

15. He C, Xu J, Zhang J et al (2012) High expression of trimethylated histone $\mathrm{H} 3$ lysine 4 is associated with poor prognosis in hepatocellular carcinoma. Hum Pathol 43(9):1425-1435. https:// doi.org/10.1016/j.humpath.2011.11.003

16. Messier TL, Gordon JA, Boyd JR et al (2016) Histone H3 lysine 4 acetylation and methylation dynamics define breast cancer subtypes. Oncotarget 7(5):5094-5109. https://doi.org/10.18632/oncot arget.6922

17. Rao RC, Dou Y (2015) Hijacked in cancer: the KMT2 (MLL) family of methyltransferases. Nat Rev Cancer 15(6):334-346. https:// doi.org/10.1038/nrc3929

18. Cancer Genome Atlas Network (2012) Comprehensive molecular portraits of human breast tumours. Nature 490(7418):61-70. https ://doi.org/10.1038/nature11412

19. Nik-Zainal S, Davies H, Staaf J, Ramakrishna M et al (2016) Landscape of somatic mutations in 560 breast cancer wholegenome sequences. Nature 534(7605):47-54. https://doi. org/10.1038/nature17676

20. Forbes SA, Beare D, Boutselakis H et al (2017) COSMIC: somatic cancer genetics at high-resolution. Nucleic Acids Res 45(D1):D777-D783. https://doi.org/10.1093/nar/gkw1121

21. Tajima K, Yae T, Javaid S et al (2015) SETD1A modulates cell cycle progression through a miRNA network that regulates p53 target genes. Nat Commun 6:8257. https://doi.org/10.1038/ncomm s9257

22. Salz T, Deng C, Pampo C et al (2015) Histone methyltransferase hSETD1A is a novel regulator of metastasis in breast cancer. Mol Cancer Res 13(3):461-469. https://doi.org/10.1158/1541-7786. MCR-14-0389

23. Ansari KI, Hussain I, Kasiri S et al (2012) HOXC10 is overexpressed in breast cancer and transcriptionally regulated by estrogen via involvement of histone methylases MLL3 and MLL4. J Mol Endocrinol 48(1):61-75. https://doi.org/10.1530/ JME-11-0078

24. Jeong KW, Kim K, Situ AJ et al (2011) Recognition of enhancer element-specific histone methylation by TIP60 in transcriptional activation. Nat Struct Mol Biol 18(12):1358-1365. https://doi. org/10.1038/nsmb.2153

25. Jeong KW, Andreu-Vieyra C, You JS et al (2014) Establishment of active chromatin structure at enhancer elements by mixed-lineage leukemia 1 to initiate estrogen-dependent gene expression. Nucleic Acids Res 42(4):2245-2256. https://doi.org/10.1093/nar/ gkt1236

26. Jozwik KM, Chernukhin I, Serandour AA et al (2016) FOXA1 directs $\mathrm{H} 3 \mathrm{~K} 4$ monomethylation at enhancers via recruitment of the methyltransferase MLL3. Cell Rep 17(10):2715-2723. https ://doi.org/10.1016/j.celrep.2016.11.028

27. Toska E, Osmanbeyoglu HU, Castel P et al (2017) PI3K pathway regulates ER-dependent transcription in breast cancer through the epigenetic regulator KMT2D. Science 355(6331):1324-1330. https://doi.org/10.1126/science.aah6893

28. Manso L, Mouron S, Tress M et al (2016) Analysis of paired primary-metastatic hormone-receptor positive breast tumors (HRPBC) uncovers potential novel drivers of hormonal resistance. PLoS ONE 11(5):e0155840. https://doi.org/10.1371/journ al.pone. 0155840

29. Kang HJ, Lee MH, Kang HL et al (2014) Differential regulation of estrogen receptor alpha expression in breast cancer cells by metastasis-associated protein 1. Cancer Res 74(5):1484-1494. https://doi.org/10.1158/0008-5472.CAN-13-2020

30. Zhang Y, Liu T, Meyer CA et al (2008) Model-based analysis of ChIP-Seq (MACS). Genome Biol 9(9):R137. https://doi. org/10.1186/gb-2008-9-9-r137

31. Robinson JT, Thorvaldsdottir H, Winckler W et al (2011) Integrative genomics viewer. Nat Biotechnol 29(1):24-26. https://doi. org/10.1038/nbt.1754 
32. Chanrion M, Negre V, Fontaine $\mathrm{H}$ et al (2008) A gene expression signature that can predict the recurrence of tamoxifen-treated primary breast cancer. Clin Cancer Res 14(6):1744-1752. https:// doi.org/10.1158/1078-0432.CCR-07-1833

33. Miller LD, Smeds J, George J et al (2005) An expression signature for p53 status in human breast cancer predicts mutation status, transcriptional effects, and patient survival. Proc Natl Acad Sci USA 102(38):13550-13555. https://doi.org/10.1073/pnas.05062 30102

34. Wang Y, Klijn JG, Zhang Y et al (2005) Gene-expression profiles to predict distant metastasis of lymph-node-negative primary breast cancer. Lancet 365(9460):671-679. https://doi.org/10.1016/ S0140-6736(05)17947-1

35. van de Vijver MJ, He YD, van't Veer LJ et al (2002) A geneexpression signature as a predictor of survival in breast cancer. $\mathrm{N}$ Engl J Med 347(25):1999-2009. https://doi.org/10.1056/NEJMo a021967

36. Kim HY, Choi JH, Lee JY et al (2019) Cancer Target Gene Screening: a web application for breast cancer target gene screening using multi-omics data analysis. Brief Bioinform. https://doi. org/10.1093/bib/bbz003

37. Schuettengruber B, Martinez AM, Iovino N et al (2011) Trithorax group proteins: switching genes on and keeping them active. Nat Rev Mol Cell Biol 12(12):799-814. https://doi.org/10.1038/ nrm3230

38. Macaluso M, Cinti C, Russo G et al (2003) pRb2/p130-E2F4/5HDAC1-SUV39H1-p300 and pRb2/p130-E2F4/5-HDAC1SUV39H1-DNMT1 multimolecular complexes mediate the transcription of estrogen receptor-alpha in breast cancer. Oncogene 22(23):3511-3517. https://doi.org/10.1038/sj.onc.1206578

39. Sharma D, Blum J, Yang X et al (2005) Release of methyl CpG binding proteins and histone deacetylase 1 from the estrogen receptor alpha (ER) promoter upon reactivation in ER-negative human breast cancer cells. Mol Endocrinol 19(7):1740-1751. https://doi.org/10.1210/me.2004-0011

40. Woodfield GW, Hitchler MJ, Chen Y et al (2009) Interaction of TFAP2C with the estrogen receptor-alpha promoter is controlled by chromatin structure. Clin Cancer Res 15(11):3672-3679. https ://doi.org/10.1158/1078-0432.CCR-08-2343
41. Flouriot G, Griffin C, Kenealy M et al (1998) Differentially expressed messenger RNA isoforms of the human estrogen receptor-alpha gene are generated by alternative splicing and promoter usage. Mol Endocrinol 12(12):1939-1954. https://doi. org/10.1210/mend.12.12.0209

42. Higuchi T, Gohno T, Nagatomo T et al (2014) Variation in use of estrogen receptor-alpha gene promoters in breast cancer compared by quantification of promoter-specific messenger RNA. Clin Breast Cancer 14(4):249-257. https://doi.org/10.1016/j. clbc.2013.10.015

43. Liu L, Kimball S, Liu H et al (2015) Genetic alterations of histone lysine methyltransferases and their significance in breast cancer. Oncotarget 6(4):2466-2482. https://doi.org/10.18632/oncotarget .2967

44. Gala K, Li Q, Sinha A et al (2018) KMT2C mediates the estrogen dependence of breast cancer through regulation of ERalpha enhancer function. Oncogene 37(34):4692-4710. https://doi. org/10.1038/s41388-018-0273-5

45. Jin ML, Kim YW, Jin HL et al (2018) Aberrant expression of SETD1A promotes survival and migration of estrogen receptor alpha-positive breast cancer cells. Int J Cancer 143(11):28712883. https://doi.org/10.1002/ijc.31853

46. Jeselsohn R, Cornwell M, Pun M et al (2017) Embryonic transcription factor SOX9 drives breast cancer endocrine resistance. Proc Natl Acad Sci USA 114(22):E4482-E4491. https://doi. org/10.1073/pnas.1620993114

47. Yu L, Liang Y, Cao X et al (2017) Identification of MYST3 as a novel epigenetic activator of ERalpha frequently amplified in breast cancer. Oncogene 36(20):2910-2918. https://doi. org/10.1038/onc.2016.433

Publisher's Note Springer Nature remains neutral with regard to jurisdictional claims in published maps and institutional affiliations. 